\title{
Research on removal of manganese in drinking water by potassium permanganate
}

\author{
Yukun Zhang ${ }^{1}$, Haishan Dong ${ }^{1, *}$, Peng Yan $^{2}$, and Xue Zheng ${ }^{1}$ \\ ${ }^{1}$ Faculty of Geosciences and Environmental Engineering, Southwest Jiaotong University, Chengdu \\ Sichuan, China \\ ${ }^{2}$ Chengdu Environmental Monitoring Center, Chengdu Sichuan, China
}

\begin{abstract}
Potassium Permanganate $\left(\mathrm{KMnO}_{4}\right)$, which is a kind of strong oxidizer, is used in water production. As $\mathrm{KMnO}_{4}$ containing Manganese, the Manganese concentration may get higher concentration by overdosing additives, however, there isn't any conclusion of the dosing interval in the present study. This paper used Manganese sand as catalyst and $\mathrm{KMnO}_{4}$ as oxidizer, trying to determine the optimal amount of the oxidizers, the dosing ratio and the dosing limit. The results showed that $\mathrm{KMnO}_{4}$ could remove manganese in drinking water effectively, when the addition ratio was 3.9, the removal efficiency of manganese reached $98 \%$.
\end{abstract}

Keywords: Drinking water; Manganese sand; Potassium permanganate; Addition ratio.

\section{Introduction}

The manganese content in groundwater in China often exceeds the standard. Manganese has a strong physiological toxicity. Excessive intake of manganese will reduce the synthesis of neurotransmitters in the brain and damage the nervous system in severe cases. ${ }^{[1]}$. Therefore, the treatment of manganese-containing drinking water is very necessary. The method of manganese removal commonly used in China is contact oxidation method, which uses the auto-catalysis of manganese sand to improve the manganese active filter membrane, and oxidizes and removes manganese through aeration or dosing of oxidant ${ }^{[2]}$. As an oxidant, potassium permanganate has also been applied and practiced in manganese removal. The chemical reaction formula is as follows:

$$
3 \mathrm{Mn}^{2+}+2 \mathrm{KMnO}_{4}+2 \mathrm{H}_{2} \mathrm{O}=5 \mathrm{MnO}_{2}+2 \mathrm{~K}^{+}+4 \mathrm{H}^{+}
$$

Some scholars used the oxidizing of potassium permanganate to oxidize divalent manganese, which also turns into divalent manganese ${ }^{[3]}$. The chemical reaction formula is as follows:

$$
5 \mathrm{Fe}^{2+}+\mathrm{KMnO}_{4}+\mathrm{H}^{+}=5 \mathrm{Fe}^{3+}+\mathrm{Mn}^{2+}+4 \mathrm{H}_{2} \mathrm{O}
$$

Studies have shown the dosage of potassium permanganate must be slightly larger than the theoretical dosage. However, although this part of the research has considered the

\footnotetext{
* Corresponding author: dhs2100@163.com
} 
problem that excessive dosage of potassium permanganate may increase the concentration of manganese instead, there is no clear upper limit for the specific dosage ${ }^{[4]}$. The innovation of this article are: Taking into account the strong adsorption of manganese sand, it is expected that the appropriate dosage of potassium permanganate should have a range. After this range, the manganese concentration in the effluent will start to rise. If the dosage continues to increase, the manganese concentration in the effluent will exceed the standard. Therefore, this experiment conducts a research on the removal of manganese by potassium permanganate contact oxidation,hoping to find the appropriate dosage of the oxidant potassium permanganate and determine the upper limit of its dosage.

\section{Materials \& method}

\subsection{Manganese water sample}

This experiment used deionized water as raw water. Since iron and manganese often exist in groundwater,the raw water for this experiment was equipped with ferrous ion with the same concentration as manganese, and quantitative manganese sulfate and ferrous sulfate stock solutions were added dropwise to the deionized water to make the concentration of iron and manganese in the raw water is $2 \mathrm{mg} / \mathrm{L}$.

\subsection{Catalyst}

The catalyst uses manganese sand, which was cleaned with deionized water to remove the floating ash and rust on the surface. Put it into a blast drying box, blast dry at $90^{\circ} \mathrm{C}$ for 6 hours, and take it out for use.

\subsection{Method}

This test used the static beaker test: We took $400 \mathrm{~mL}$ of iron-manganese-containing raw water into a $500 \mathrm{~mL}$ beaker, added the catalyst, and placed it on a six-joint mixer. We added an appropriate amount of oxidant to stir and started timing. The stirring speed was 300 $\mathrm{r} / \mathrm{min}$. We regularly took the supernatant for determination to determine the best reaction time and the best oxidant dosage.

The concentration of manganese ions in this experiment was measured by a flame atomic absorption spectrophotometer.

We did two sets of parallel experiments according to the above experimental steps to ensure the accuracy of the experimental results.

The chemical reagents used in the experiment are shown in Table 1 below.

Table 1. The main chemical reagents used in the experiment.

\begin{tabular}{|c|c|c|c|}
\hline Number & Reagent name & Specification & Manufacturer \\
\hline 1 & $\mathrm{MnSO}_{4} \cdot 4 \mathrm{H}_{2} \mathrm{O}$ & $99 \%$ & $\begin{array}{c}\text { Chengdu Jinshan Chemical } \\
\text { Reagent Co., Ltd. }\end{array}$ \\
\hline 2 & $\mathrm{FeSO}_{4} \cdot 7 \mathrm{H}_{2} \mathrm{O}$ & $99 \% \sim 101 \%$ & $\begin{array}{c}\text { Chengdu Jinshan Chemical } \\
\text { Reagent Co., Ltd. }\end{array}$ \\
\hline 3 & $\mathrm{KMnO}_{4}$ & $\mathrm{AR}$ & $\begin{array}{c}\text { Chengdu Jinshan Chemical } \\
\text { Reagent Co., Ltd. }\end{array}$ \\
\hline 4 & $\begin{array}{c}\text { Manganese } \\
\text { sand }\end{array}$ & $\begin{array}{c}0.8 \sim 2 \mathrm{~mm}(\mathrm{Par} \\
\text { ticle size) }\end{array}$ & $\begin{array}{c}\text { Gongyi Hengxin Filter } \\
\text { Material Factory }\end{array}$ \\
\hline
\end{tabular}




\section{Results \& discussion}

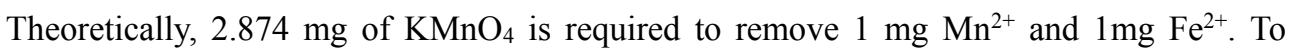
ensure that $\mathrm{Mn}^{2+}$ in the water can be effectively removed, the amount of potassium permanganate added should satisfy $\left[\mathrm{KMnO}_{4}\right] /\left[\mathrm{Mn}^{2+}\right]\left[\mathrm{Fe}^{2+}\right]>2.874$, taking into account manganese sand has a certain adsorption effect on iron and manganese, and the dosage of low potassium permanganate should be considered in the test.Therefore, the test first determined the general scope of application of potassium permanganate. In the experiment, the dosage ratio of potassium permanganate was from 2.5 to 5 , the $\mathrm{pH}$ value of the raw water was adjusted to 7.5, the reaction time was $40 \mathrm{~min}$, and the removal effect was as shown in the figure below.

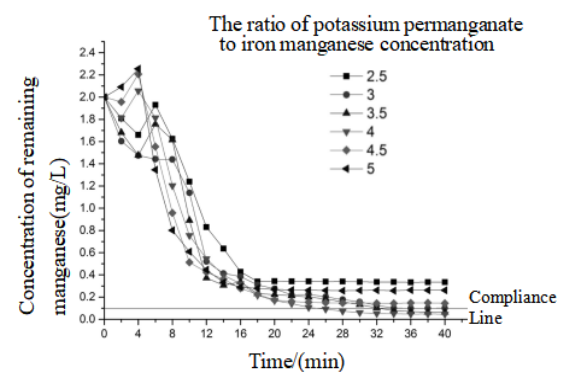

Fig. 1. The remaining concentration of manganese with a dosing ratio between 2.5 and 5 .

The manganese concentration in the raw water was $2 \mathrm{mg} / \mathrm{L}$, which means that to reach the standard value of $0.1 \mathrm{mg} / \mathrm{L}$, a $95 \%$ removal rate was required. From Figure 1 and Figure 2 , it can be seen that when the potassium permanganate dosage ratio was less than the theoretical dosage ratio, the removal effect of manganese was not good, only $83.3 \%$, and the reaction balanced at about $20 \mathrm{~min}$. When the dosing ratio exceeded the theoretical dosing amount, the removal efficiency of manganese increased.

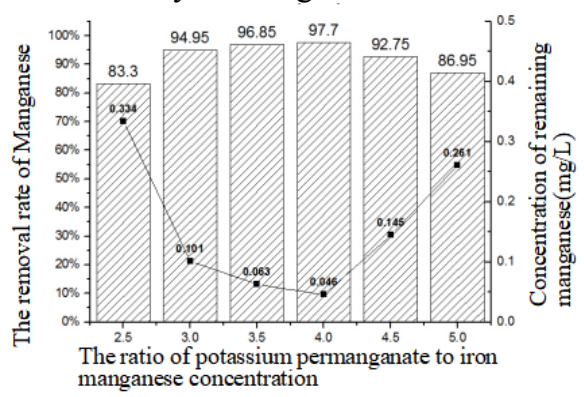

Fig. 2. The final removal effect of manganese with a dosing ratio between 2.5 and 5 .

When the dosing ratio was between 3 and 4, the manganese concentration in the effluent water was significantly reduced. Among them, when the dosing ratio was 3 , the manganese concentration in the effluent water was already close to the standard. When the concentration was 3.5 or 4 , the manganese concentration in the effluent had reached the standard. When the reaction was completed, there were more suspended solids in the water, which was brown, and the effluent needed to be filtered, and the effluent was transparent. The reaction balanced after $30 \sim 35 \mathrm{~min}$, indicating that potassium permanganate fully reacted with iron and manganese, the efficiency of manganese removal was the highest, and the best dosing ratio was between 3.5 4. When the dosing ratio exceeded 4 and was between 4.5 and 5, the manganese concentration in the effluent increased instead, and the effluent appeared pale pink, indicating that potassium permanganate had been dosed too 
much, and some potassium permanganate had not reacted, and the reaction equilibrium time was relatively short. It was speed up before and reached equilibrium in $16 \sim 20 \mathrm{~min}$. This is because the increase of potassium permanganate concentration speeded up the reaction speed, which made the manganese in the raw water faster to remove, but the remaining potassium permanganate made the effluent water worse. It can be seen from Figure 1 that at the beginning of the reaction, the concentration of manganese decreased with time due to the oxidation of manganese in the raw water by potassium permanganate, resulting in the formation of $\mathrm{MnO}_{2}$; between $4 \mathrm{~min}$ and $10 \mathrm{~min}$, The concentration of manganese had risen to varying degrees. This was because potassium permanganate first reacted with iron. During the reaction, it was deoxidated to divalent manganese, which increased the concentration of manganese in the water. The monitoring of iron also showed that within $10 \mathrm{~min}$, most of the iron in the raw water had been removed, and the iron concentration in the effluent had reached trace amounts within $20 \mathrm{~min}$. And the filtered water was still pale pink. After that, potassium permanganate oxidized the $\mathrm{Mn}^{2+}$ remaining in the raw water and the $\mathrm{Mn}^{2+}$ generated in the reaction with iron, and removed it. The reaction was completed within $30 \mathrm{~min}$ to $35 \mathrm{~min}$.

It can be seen from Figure 2 that the removal rate of manganese was the highest when the dosing ratio was 3.5 and 4 , and when the dosing ratio was 4.5 , the removal effect decreased instead, indicating that the best dosing ratio of potassium permanganate should be 3.5 4. In order to find the best dosing ratio of potassium permanganate, it was necessary to continue the test in the interval of 3.5 4 dosing ratio. At the same time, because the effluent manganese concentration exceeded the standard when the dosing ratio was 4.5, in order to find the upper limit of potassium permanganate dosing, it was necessary to continue the test between the dosing ratio of 4 to 4.5 . Therefore, we continued to test in this interval, adjusted the $\mathrm{pH}$ to 7.5 , and dosed potassium permanganate according to the dosing ratio of 3.6, 3.7, 3.8, 3.9, 4.1, 4.2, 4.3, and 4.4, and sampled after 40 minutes of reaction. Figure 3 showed the final removal effect of manganese at a dosing ratio of 3.5 to 4.5 .

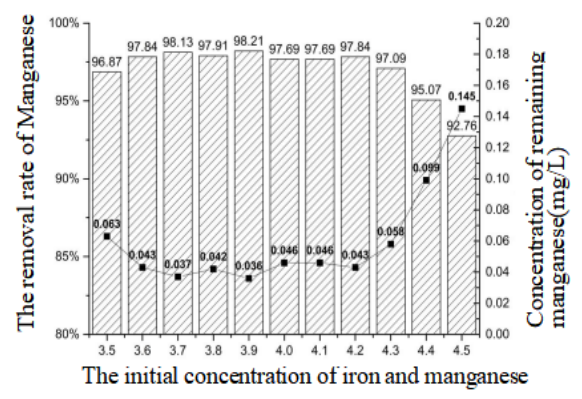

Fig. 3. The final removal effect of manganese with a dosing ratio between 3.5 and 4.5.

It can be seen from Figure 3 that when the dosing ratio was 3.9, the removal efficiency of manganese was the highest, reaching $98.21 \%$, the manganese concentration in the effluent was $0.036 \mathrm{mg} / \mathrm{L}$, and the removal efficiency was extremely good. When the dosing ratio was between 3.6 and 4.2, the removal effect of manganese was relatively balanced, and the removal efficiency was about $98 \%$, which was also a optimal dosing ratio of potassium permanganate for manganese removal. When the dosing ratio was higher than 4.3, although the effluent manganese concentration reached the standard, but the removal efficiency decreased significantly. The unreacted potassium permanganate concentration increased, which easily caused the effluent manganese concentration to increase instead, making the effluent light pink. In addition, the remaining potassium permanganate was likely to further oxidized the oxidized tetravalent manganese to generate hexavalent manganese that was soluble in water, that was, $\mathrm{MnO}_{4}{ }^{2-}$, which caused the reverse 
dissolution of manganese. Therefore, the upper limit of potassium permanganate dosage should be strictly controlled, and its dosage ratio should be less than 4.3. Dosing potassium permanganate with a dosing ratio of 3.6 to 4.2 can effectively remove manganese in the water and make the effluent reach the standard. It is a relatively safe treatment to control the dosing ratio at about 3.9.

\section{Conclusion}

- Potassium permanganate can effectively oxidize manganese ions in the water to reduce its concentration. When the dosing ratio is between 3.6 and 4.2, the manganese concentration in the effluent is very low and can reach trace amounts.

- Excessive dosage of potassium permanganate will increase the manganese concentration in the effluent. In the operation, the dosage of potassium permanganate should be strictly controlled. When the dosage ratio is 4.5 in the test, the manganese concentration in the effluent has exceeded the standard. Controlling the ratio at around 3.9 is a relatively safe way to deal with it.

- When iron exists the same time, potassium permanganate will oxidize the iron first, and the oxidation rate is very fast. In the experiment, it can be seen that the manganese concentration will increase in the first $10 \mathrm{~min}$, and sometimes even exceed its initial concentration. This is because potassium permanganate will oxidizes the iron itself and is reduced to divalent manganese ions.

\section{References}

1. XUE Xiu qin, ZHANG Rui juan. Current Status and Future Research of Manganese Health Research.J.Foreign Medical Sciences(Section of Medgeography), 29(1), 44-46 (2008)

2. SHI Zhen nan, CAI Zhen yu, LI Simin, YIN Lian feng, ZHANG Zhigang. Productive usage of permanganate removing manganese in surfacewater plant in Handan.J. Journal of Hebei Institute of Architectural Science and Technology, 21(2), 9-11 (2004)

3. Aschner J L C. Manganese neurotoxicity:cellular effects and bloodbrain barriertransport[J]. Neurose Biobeha V Rev, 15, 333-340 (2017)

4. Guo Y M, Huang T L, Wen G. The Simultaneous removal of ammonium and manganese from groundwater by iron-manganese co-oxide filter film: The role of chemical catalytic oxidation for ammonium removal[J]. Chemical Engineering Journal, 308, 322-329 (2017)

5. Bertone E, Stewart R A, Zhang H, et al. Statistical Analysis and Modelling of the Manganese Cycle in the Subtropical Advancetown Lake, Australia[J]. Journal of Hydrology: Kegional Studies, 8, 69-81 (2016)

6. Gerke T L, Little B J, Barry Maynard J. Manganese Deposition in Drinking Water Distribution Systems[J]. Science of the Total Environment. 541, 184-193 (2016)

7. Qingfeng Cheng, Yang Huang, Lichao Nengzi, et al. Performance and Microbial Community Profiles in Pilot-scale Biofilter for Ammonia,Iron and Manganese Removal at Different Dissolved Oxygen Concentrations[J]. World Journal of Microbiology and Biotechnology, 35(3), 1-11 (2019)

8. Wenbo Liu, Alette A. M. Langenhoff, Nora B. Sutton, et al. Biological Regeneration of Manganese(IV)and Iron(III)for Anaerobic Metal Oxide-mediated Removal of Pharmaceuticals from Water[J]. Chemosphere, 28, 122-130 (2018) 